\section{CLASSIFICATION OF RHEOLOGICAL PROPERTIES}

A T a meeting of the British Rheologists' Club held $A$ at the Imperial College of Science, London, on May 16, the accompanying chart was presented for discussion by the Committee. The discussion was introduced by Dr. V. G. W. Harrison (hon. treasurer).

It is believed that this classification marks a considerable advance on the earlier draft discussed at Birmingham, which was reproduced in NATURE of Feb. 14, p. 197. It incorporates many of the suggestions made by members at that meeting, and also the hatched 'frame' enclosing those categories for which the rate of deformation is liable to increase or decrease with time for constant stress is eliminated. The Committee realized that the use of this frame as possible, examples of materials showing each type of deformation should be quoted. Mr. J. C. Gage doubted whether the insertion of the graphs had adequately replaced the 'frame' of the earlier table.

Dr. J. Needham asked whether the complex rheological properties described by him in a paper read earlier in the day could be fitted into the table, and Mr. L. Bilmes submitted a circular form of the table, which the Committee will consider in the near future.

Replying on behalf of the Committee, Dr. G. W. Scott Blair (hon. secretary) said that he did not think that it would be possible to explain all kinds of complex rheological behaviour in terms of the present table. The Committee has taken as its first task the formulating of a table of types of deformation. It is hoped that with the help of members and

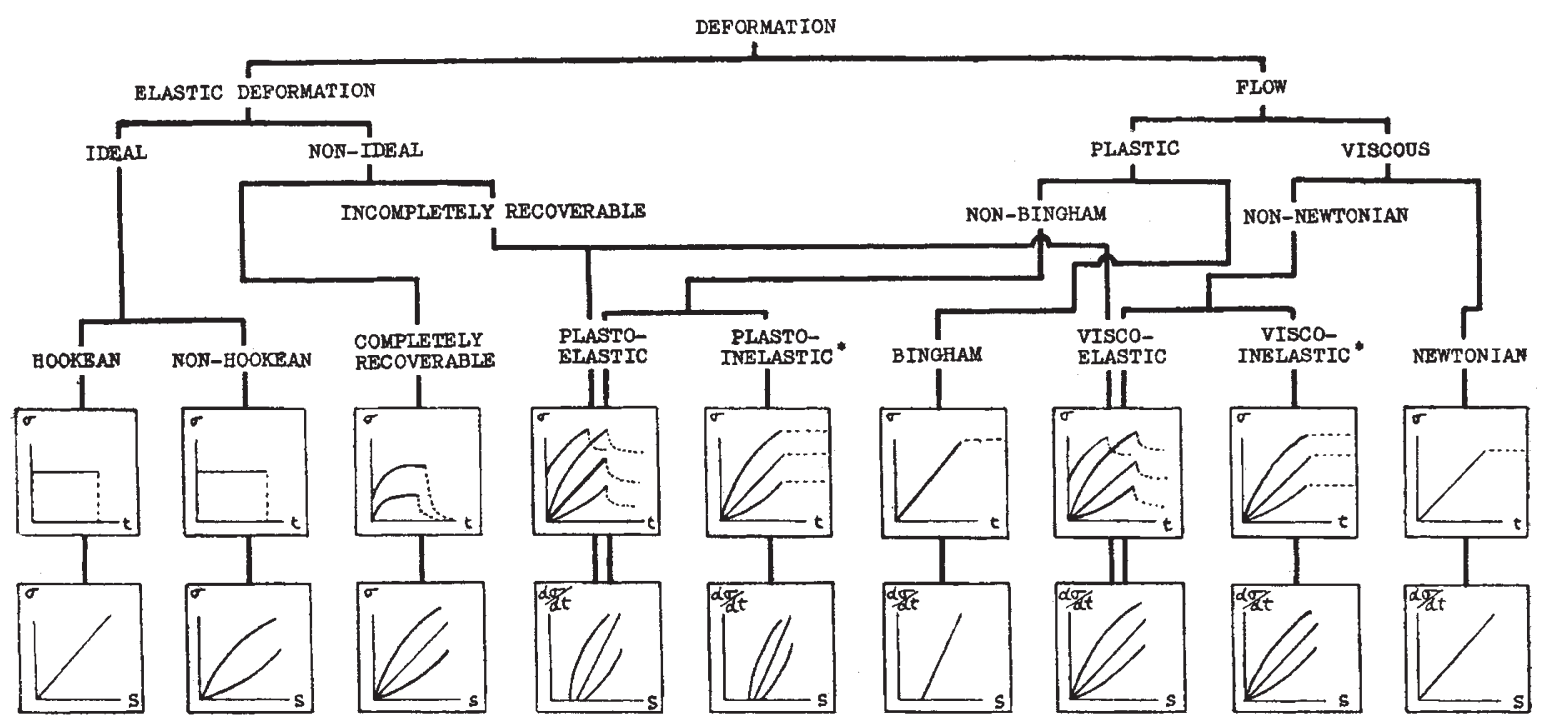

(1) o represents shear strain. $S$, " stress. $t \quad$, time.

(2) Continuous curves represent deformation under stress.

(3) Dotted curves represent behaviour after removal of stress.

(4) Where two or more curves appear in a diagram, these indica te alternative types of behaviour.

added considerably to the complexity of the table and that, even with it, the picture was incomplete, since another 'frame' would be required to allow for changes in the strain $(\sigma)-$ time $(t)$ relation with changes in stress $(S)$.

In order to simplify the table, Mr. D. C. Broome had been asked to draw graphs relating $\sigma / t, \sigma / S$ and $(d \sigma / d t) / S$ for each category showing, where necessary, the possible types of curvature which may occur in each category. These graphs, after slight revision by the Committee, have been included in the revised table. The table has also been re-arranged so that elastic deformations remain on the left-hand side throughout, the viscous and plastic deformations being on the right.

In the discussion which followed, Dr. C. A. Maunder Foster suggested that the Committee might proceed to consider the more detailed drawing of recovery curves and Mr. J. Pryce-Jones proposed that, as soon
(5) In cases marked * either the $\sigma / t$ curve or the $\frac{d \sigma}{d t} / S$ curve may be a straight line, but not both, since in this case the systems would be Bingham or"Newtonian, which are given separate categories.

(6) The upper series of diagrams in which shear strain is plotted against time represents deformations under constant stress

The lower series, in which $d a / d t$ is.plotted against stress, is for an arbitrary time.

of colleagues in the (American) Society of Rheology, the scope of classification of properties may be extended, but for the present the effective range of activity is limited. It is realized that the categories in the table cannot be regarded as hard-and-fast limits of behaviour, but rather as convenient landmarks, analogous in many ways to the psychologist's classifications of character and personality types. It would be rash to regard intermediate systems as, of necessity, mechanical mixtures of the simple types between which they lie. As soon as the categories are found to be acceptable, the Committee will consider the possibility of designating each type by means of a letter or number.

Any one who may wish to make criticisms or suggestions with regard to the above table is invited to communicate with the honorary secretary of the British Rheologists' Club, c/o Institute of Physics, University of Reading. 